\title{
Décadrages Décadrages
}

cinéma, à travers champs Cinéma, à travers champs

37-38 | 2018

Lionel Rogosin

Compte rendu d'Eric Rohmer : le spectateur séduit. De la représentation de Maria Tortajada, Paris, Kimé, 2017

Achilleas Papakonstantis

\section{OpenEdition}

Journals

Édition électronique

URL : https://journals.openedition.org/decadrages/1291

DOI : 10.4000/decadrages.1291

ISSN : 2297-5977

Éditeur

Association Décadrages

Édition imprimée

Date de publication : 1 mars 2018

Pagination : 207-213

ISBN : 978-2-9700963-4-4

ISSN : 2235-7823

Référence électronique

Achilleas Papakonstantis, "Compte rendu d'Eric Rohmer : le spectateur séduit. De la représentation de Maria Tortajada, Paris, Kimé, 2017 », Décadrages [En ligne], 37-38 | 2018, mis en ligne le 02 février 2021, consulté le 04 avril 2022. URL : http://journals.openedition.org/decadrages/1291 ; DOI : https:// doi.org/10.4000/decadrages.1291 
Compte rendu d'Eric Rohmer: le spectateur séduit. De la représentation de Maria Tortajada, Paris, Kimé, 2017

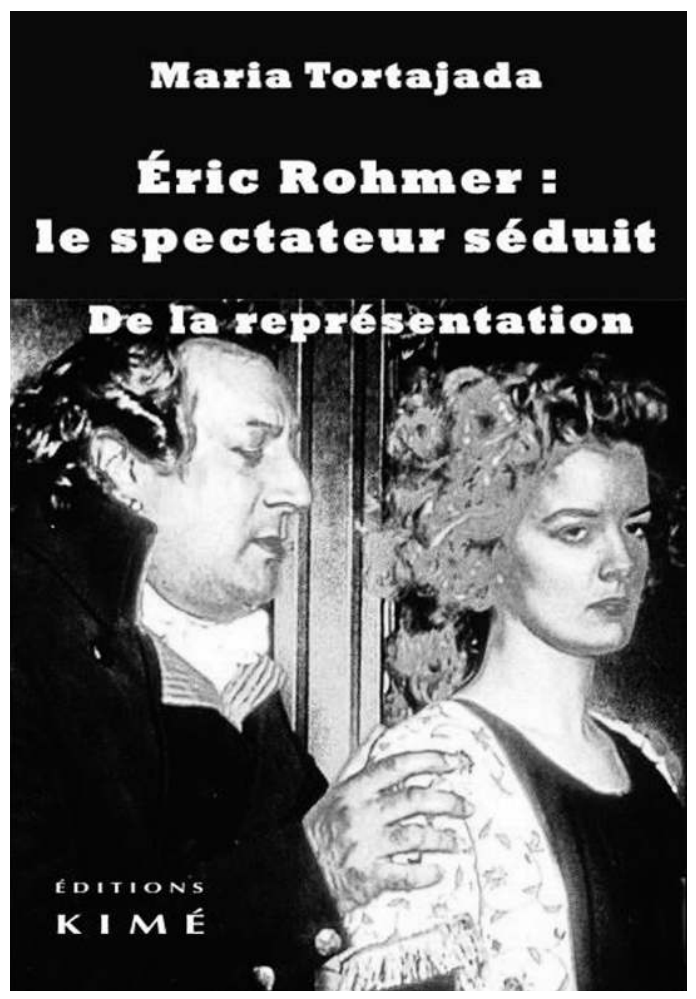

IL N'EST GUÈRE SURPRENANT, depuis l'annonce de la disparition d'Eric Rohmer en 20I0, qu'un grand nombre de publications portant sur sa vie (par exemple la biographie signée par Antoine de Baecque et Noël Herpe ${ }^{1}$ ) et son ouvre (Rohmer en perspectives et Le conteur du cinéma ${ }^{2}$, parmi d'autres) aient vu le jour. Il semblerait alors que l'ouvrage de Maria Tortajada, professeure à l'Université de Lausanne, vienne trouver sa place au sein de cette prolifération éditoriale. Or, Eric Rohmer: le
1 Antoine de Baecque, Noël Herpe, Eric Rohmer. Biographie, Paris, Stock, 2014 .

2 Silvie Robic, Laurence Schifano (éd.), Rohmer en perspectives, Paris, Presses Universitaires de Paris Ouest, 2013; Haydée Caillot, Eric Rohmer. Le conteur du cinéma, Paris, A dos d'âne, 2013. Ajoutons un exemple issu du contexte anglophone: Vittorio Hösle, Eric Rohmer. Filmmaker and Philosopher, Londres/ New York, Bloomsbury, 2016. 
3 A première vue légère mais significative par rapport aux intentions structurant le nouveau projet de Tortajada, la modification du titre mérite d'être soulignée: le livre de I999 s'intitulait Le spectateur séduit. Le libertinage dans le cinéma d'Eric Rohmer.

4 Par ailleurs, en développant son hypothèse topographique, Tortajada établit, en toute connaissance de cause, la «mise en scène»- notion-clé pour l'équipe des Cahiers - comme une valeur constitutive de la qualité d'auteur de Rohmer. spectateur séduit. De la représentation se distingue d'emblée des autres monographies consacrées au cinéaste, et ceci doublement.

Premièrement, il s'agit d’une réédition considérablement augmentée d'un livre - couronné du Prix Barbour de critique littéraire (Université de Genève) et aujourd'hui épuisé - paru chez le même éditeur en $1999^{3}$, du vivant de Rohmer. En effet, l'actualité rohmérienne était particulièrement dense dans les années 2000: en plus de la sortie en salles de trois nouveaux longs métrages, le cinéaste avait également pris soin de déposer, peu avant sa mort, ses propres archives à l'Institut Mémoires de l'édition contemporaine (IMEC), ouvrant ainsi l'accès à un corpus riche et hétérogène de textes inédits. Ces nouvelles données ont confirmé les thèses de la première édition, comme en attestent les analyses proposées dans la seconde. En même temps, elles ont offert la possibilité d'un élargissement du modèle théorique développé par Tortajada, notamment en ce qui concerne l'exploration des limites entre cinéma et littérature, justifiant alors l'entreprise d'une réédition.

Deuxièmement, Le spectateur séduit n'est pas un livre sur Rohmer à proprement parler. Certes, Tortajada participe - de manière consciente, comme les premières pages de l'introduction en attestent - à la construction discursive du cinéaste en figure d'auteur; qui plus est, elle le fait en respectant un des principes fondamentaux de la politique des auteurs telle qu'elle fut déployée par les critiques des Cahiers du cinéma (dont Rohmer) dans les années I950, à savoir l'«unité de style ${ }^{4}$. Elle procède pourtant en contradiction avec les prises de position de l'auteur. Alors que Rohmer rejette ouvertement la référence à la littérature libertine, Tortajada refuse de le croire sur parole. D’après elle, le libertinage constitue la force structurante du récit rohmérien, capable d'expliquer à la fois les rapports entre les personnages de l'univers diégétique et le lien que les films tissent avec le spectateur. En d'autres termes, et ceci constitue un des postulats de base de la méthodologie de Tortajada, ce n'est pas en suivant à la lettre les déclarations du cinéaste que l'unité de son style émerge - comme l'aurait prétendu un certain auteurisme simpliste mais à travers des analyses minutieuses de l'œuvre filmée et écrite qu’il nous a léguée.

Si ces analyses sont à compter parmi les points les plus forts du livre, c'est parce qu'en plus de leur valeur argumentative et de la finesse d'écriture dont elles témoignent, elles se trouvent mises au service d'une étude 
théorique à part entière. Beaucoup plus qu'une mise au point générale sur le cinéma rohmérien, Le spectateur séduit se révèle comme le «site» du déploiement par Tortajada d'une théorie de la représentation au cinéma. Articulée autour d'une certaine acception de la notion de séduction, cette théorie constitue la proposition centrale du livre et mérite ainsi d'être synthétisée et expliquée ici.

Le modèle de Tortajada se base sur un schéma tripartite capable de résumer tout rapport de tenant lieu: représenté - représentation - spectateur. Or, ce livre renonce dès le départ à l'examen séparé de chacun de ces termes ${ }^{5}$; l'analyse portera sur leurs interactions, sur le réseau des relations qui les lie et les intègre à ce qu'on pourrait désigner comme un dispositif représentationnel. Il sera ainsi question des rapports variables entre la représentation et ce qu'elle donne à voir (parcourant toute la distance qui va de la transparence de la «vitre» au leurre), et surtout des effets visés sur le destinataire de cette représentation, le spectateur, dont l'importance primordiale au sein de cette étude se trouve affirmée déjà par le titre du livre. S’il est vrai que le schéma exposé ci-dessus caractérise tous les films (comme d'ailleurs les tableaux, les photographies, voire toute œuvre figurative), la particularité du cinéma de Rohmer, selon l'auteure, est qu'il propose dans son texte même ${ }^{6}$ une théorisation des rapports de représentation à travers la référence à la littérature libertine. Des précisions supplémentaires sont ici nécessaires.

Au départ de l'argumentaire de Tortajada se trouve le constat suivant: la séduction constitue le sujet central des films et des écrits de Rohmer, caractéristique qu'ils partagent avec les grands romans libertins du $\mathrm{XVIII}^{\mathrm{e}}$ siècle. Le premier chapitre propose alors de cerner le réseau sémantique de la notion de séduction afin d'éclairer ses définitions souvent contradictoires. A l'issue de ce travail de conceptualisation, le lecteur se trouve convaincu de la pertinence d'une définition représentationnelle du terme, la seule capable de réunir toutes les variantes de la séduction: on a toujours affaire à un spectacle, une mise en scène, un tenant lieu renvoyant au désir (réel ou feint) du séducteur et s'adressant à un spectateur. Appréhender la séduction comme une mise en représentation du désir permet à Tortajada d'élargir son propos, de dépasser l'analyse interne des films (et plus particulièrement des rapports de séduction entre les personnages) afin d'éclairer les stratégies de la représentation filmique visant, à leur tour, à séduire le spectateur.
5 Un tel examen aurait nécessité une incursion dans le champ de la philosophie pour préciser, par exemple, la nature du «représenté» (le monde réel ou celui des idées, les phénomènes ou les essences, etc.), mais aussi dans celui de la sociologie afin de dresser le portrait des spectateurs des films de Rohmer au fil des décennies.

6 Par «texte», il nous faut ici entendre à la fois les dimensions narratives et formelles des films de Rohmer. 
7 Reconnaissant l'hétérogénéité du corpus libertin, comme d'ailleurs le caractère fluctuant de l'étiquette «littérature libertine», Tortajada précise plus d'une fois qu'elle se concentre pour sa part sur la voie tracée par ces deux auteurs célèbres.

8 En lisant le premier chapitre, on apprend que l'opposition entre transparence et leurre - classique dans les débats artistiques autour du réalisme - se retrouve dans les discours portant sur la séduction. Selon Tortajada, la séduction par l'ambiguïté serait alors subversive car «elle ne permet pas au garant de la représentation de s'installer dans une vérité devant une figuration fermée et définitive» (p. 20).

9 La confrontation du modèle de Tortajada aux théories réalistes devient explicite dès le début du livre. Le nom d’André Bazin apparaît dans sa toute première phrase, comme d'ailleurs le terme «réalisme». Ce dernier revient régulièrement dans l'introduction, qualifiant chaque fois le cinéma de Rohmer.
De toutes les formes possibles de la séduction, Tortajada retient celle qui se base sur l'ambiguïté, jugée comme centrale dans l'œuvre de Rohmer et plus à même d'éclairer le fonctionnement du dispositif représentationnel par le doute irréductible dans lequel elle plonge son destinataire: ce dernier est à la fois confronté à l'ambiguïté du désir de l'autre et ramené à l'ambiguïté de son propre statut face à la représentation séduisante. Ainsi, partant de l'analyse du comportement des personnages aussi bien dans les films et les textes de Rohmer que dans les écrits de Crébillon fils et de Laclos ${ }^{7}$, l'auteure construit un modèle anthropologique qui sera par la suite transposé sur un plan esthétique: la séduction par l'ambiguïté élevée au rang d'une théorie de la représentation au sein de laquelle la place du spectateur est en jeu. C’est sur ce point précis que le modèle théorique de Tortajada s'articule avec les débats sur le réalisme qui font florès après la Seconde Guerre mondiale ${ }^{8}$. La notion même d'ambiguïté ne peut que le rapprocher de la théorie réaliste d’André Bazin, défendue d'ailleurs par Rohmer lui-même 9 . Car Tortajada n'appréhende pas le réalisme bazinien selon les termes de la transparence, mais plutôt comme la mise en place d'une représentation ambiguë - l'analyse de la séquence finale du Rayon vert (1986) dans le troisième chapitre en offre une explicitation éloquente. La séduction par l'ambiguïté et le réalisme bazinien ne doivent pas être confondus, précise-t-elle; cependant, en introduisant la «tercéité» comme élément crucial de la représentation, ces deux modèles constituent des alternatives au dualisme représentationnel auquel adhéraient les défenseurs du réalisme, tout comme ses critiques le plus féroces. Dans ce cas, alors, le spectateur n'est ni leurré ni démystifié, mais séduit car confronté à sa propre ambiguïté, pris dans une position de tiers instable.

Afin de mieux expliquer la place du spectateur comme un «tiers» dans la théorie de Tortajada, il est nécessaire de se pencher de plus près sur son analyse de la séduction par l'ambiguïté. Cette dernière se décline en deux modèles distincts: d'un côté, une version sémiologique, à savoir le dédoublement du langage et le jeu du double sens qui condamne le destinataire à une indétermination interprétative face à la représentation; de l'autre, une version topographique qui voit le sujet séduit parce que contraint d'occuper simultanément deux places incompatibles (à la fois à l'intérieur du rapport de séduction et à l'extérieur, faisant office de témoin). L'articulation de ces deux modèles constituerait ainsi le «principe moteur du cinéma de Rohmer» (p. I07). 
Si la rhétorique des doubles registres, maîtrisée par les grands libertins du XVIII ${ }^{\mathrm{e}}$ siècle, a été maintes fois analysée par les spécialistes du champ littéraire, l'hypothèse topographique émerge comme une des thèses originales du Spectateur séduit. Elle est déjà présentée à la fin du premier chapitre à travers l'analyse d'un texte inédit, datant de 1943, intitulé «Fin de journée», et du scénario du court métrage Présentation, publié en 1952 dans les Cahiers du cinéma. Dans les deux cas, les personnages principaux se trouvent, malgré eux, captés dans une oscillation imaginaire entre deux positions incompatibles (intérieure-extérieure) ${ }^{10}$. Ils participent pleinement au jeu de la séduction mais, à force de vouloir être aussi en position de spectateur capable d'évaluer le déroulement du jeu, ils se transforment paradoxalement en sujets séduits. La démarche de Tortajada, développée dans le deuxième chapitre, consiste à examiner le potentiel réflexif de la situation de ces personnages par rapport au fonctionnement de la représentation filmique et ses effets sur le spectateur.

Pour ce faire, elle analyse dans un premier temps le rôle du tiers garant dans la littérature libertine, référence capable d'expliquer la séduction par l'ambiguïté telle qu’elle se développe dans le cinéma de Rohmer. Ce tiers est le public de la société aristocratique du XVIII ${ }^{\mathrm{e}}$ siècle: un «grand œil» prêt à réprimander au nom de la bienséance mais aussi le point central à partir duquel s'orchestre le jeu de la séduction. «S”il est garant de la loi, le public est aussi à l'origine du dédoublement, de l'essence même de la séduction. Le modèle libertin définit sa place explicitement: il est ce tiers que les personnages ne cessent de mentionner, qui sature de sa présence l'espace social et qui reste particulièrement stable malgré son statut paradoxal» (p. 159). Dans le récit rohmérien, il n’y a aucune référence, bien évidemment, au public. Selon un schéma mis en place très tôt dans l'œuvre filmique de Rohmer, dans la série des Contes moraux, la place du tiers garant est revendiquée par le hérosnarrateur: au début du film, il énonce les règles du «jeu» (sa morale) pour se poser par la suite garant de son propre récit. Or, il se trouve de cette manière dans une position intenable car «il est simultanément celui qui détient et défend la loi et celui qui est censé la contourner. Il assume à la fois, dans la constellation libertine revue par Rohmer, deux places incompatibles» (p. I6o).

Par ailleurs, à la différence d'un Versac ou d'un Valmont, les héros rohmériens renoncent à la finalité du jeu de la séduction, l’acte érotique,
10 A propos du protagoniste de Présentation: «Si, pour savoir, la meilleure situation est celle qui le rend extérieur, pour séduire, il doit se trouver au milieu d'elles [Alice et Clara, les deux personnages féminins du film]» (pp. I04-I05). 
11 C’est précisément sur ce rapport problématique des héros à l'action que Tortajada repère le «modernisme» du récit rohmérien. Le passage par la référence libertine expliquerait ainsi ce grand paradoxe du cinéma de Rohmer, situé le plus souvent par le discours critique aux croisements du classicisme et du modernisme.

12 Selon la célèbre formulation metzienne, «c'est moi [le spectateur] qui fais le film» (Christian Metz, Le Signifiant imaginaire, Paris, Bourgois, I984, p.69, cité par M. Tortajada, p. I7I). en lui substituant la réévaluation de ce renoncement par la parole " . «Les Contes moraux déplacent pour leur part le lieu de l'interrogation. Ce n'est pas dans l'action que se risque le sujet mais ailleurs, dans la place du tiers garant qu'il prétend adopter. Les films problématisent la position du sujet lui-même quand celui-ci se présente comme témoin, spectateur de son acte, alors tiers instable, sujet séduit» (p. ${ }_{5} 6$ 6). Par le biais d'une analyse de La Boulangère de Monceau (ig62), Tortajada déplace l'interrogation des personnages-spectateurs au destinataire de la représentation filmique, le spectateur $d u$ film.

Si ce dernier incarne, selon Christian Metz, la condition de possibilité du signifiant filmique ${ }^{12}$, Tortajada nous rappelle que c'est «le modèle du spectateur garant, centre organisateur de la représentation filmique qui est postulé comme principe du cinéma classique» (p. I7I). Au sein du système qui instaure le spectateur comme origine de la représentation, on distinguerait alors deux modes de participation à la fiction. Soit le spectateur occupe une place à l'intérieur de la diégèse grâce à son identification à la caméra, tout en restant résolument extérieur aux aventures racontées, c'est-à-dire en position de garant privilégié, soit il se trouve absorbé par l'histoire en s'identifiant pleinement aux personnages à l'écran. Si tous les films peuvent alterner entre ces deux modes en fonction de l'effet recherché, le cinéma de Rohmer vise explicitement, selon Tortajada, à créer des moments d'ambiguïté autour de cette question de la participation spectatorielle. Ses films empêchent, à des moments précis, de tenir de manière certaine l'une ou l'autre de ces positions incompatibles. Selon les préceptes de la séduction par l'ambiguïté, le spectateur se trouve ainsi transformé en tiers instable et, par conséquent, séduit. Au moment même où il pouvait se croire rassuré par une histoire aux apparences classiques, voire même naïves, il se retrouve finalement déstabilisé par un processus d'indétermination. Dans son troisième et dernier chapitre, le Spectateur séduit propose une série d'analyses de séquences visant à expliciter la réappropriation rohmérienne des topoï du récit de la séduction, tels que le coup de foudre ou le croisement des regards au miroir; «contaminés» eux-mêmes comme figures par le principe de l'ambiguïté, ils offrent encore une mise en abyme du fonctionnement de la représentation filmique chez Rohmer.

Forcément elliptique, une brève synthèse du Spectateur séduit, telle que nous l'avons tentée ici, ne saurait prétendre rendre compte de tous 
les aspects et ramifications des thèses avancées dans ses pages. La construction théorique de Tortajada est étayée sur une partie analytique extrêmement riche et rigoureuse, d'une écriture passionnante, parfois lyrique mais toujours très claire. Arrivé à la fin de cet ouvrage, le lecteur sera en mesure de poser un regard neuf, non seulement sur le cinéma d'Eric Rohmer, mais aussi sur sa propre place de spectateur face aux différents dispositifs de représentation qui l'entourent et l'incluent. 